\title{
TRANSPOSABLE AND SYMMETRIZABLE MATRICES
}

\author{
DAVID MCCARTHY and BRENDAN D. MCKAY
}

(Received 3 December 1979)

Communicated by W. D. Wallis

\begin{abstract}
A square matrix $A$ is transposable if $P(R A)=(R A)^{\mathrm{T}}$ for some permutation matrices $P$ and $R$, and symmetrizable if $(S A)^{\mathrm{T}}=S A$ for some permutation matrix $S$. In this paper we find necessary and sufficient conditions on a permutation matrix $P$ so that $A$ is always symmetrizable if $P(R A)=(R A)^{\mathrm{T}}$ for some permutation matrix $R$.
\end{abstract}

1980 Mathematics subject classification (Amer. Math. Soc.) : 05 B 20, 05 C 25, 05 C 50.

\section{Introduction}

A square matrix $A$ is transposable if $Q A R=A^{\mathrm{T}}$ for some permutation matrices $Q$ and $R$, and symmetrizable if $(S A)^{\mathrm{T}}=S A$ for some permutation matrix $S$.

Clearly all symmetrizable matrices are transposable, since $(S A)^{\mathrm{T}}=S A$ implies $S A S=A^{\mathrm{T}}$. In this paper we determine sufficient conditions for a transposable matrix to be symmetrizable, and prove that these conditions, in a weaker sense to be described, are also necessary.

Of particular interest are the binary matrices $A$, especially when $A$ is the incidence matrix of a symmetric balanced incomplete block design (SBIBD). In this case if $A$ is transposable then the SBIBD corresponding to $A$ is self-dual, or equivalently the SBIBD admits a correlation. If $A$ is a symmetrizable then the SBIBD is self-polar, or equivalently admits a correlation of order 2 , a polarity. Definitions of the above terms can be found in Dembowski (1968).

Self-polar designs are of interest since one can obtain a graph from these designs by taking a symmetric form of the incidence matrix and interpreting it as the adjacency matrix of a graph. These graphs may contain loops. If the parameters of the self-polar SBIBD are $(v, k, \lambda)$ then we call the graph a $(v, k, \lambda)$-graph. Such a graph is a $k$-regular graph on $v$ vertices with the property that any two vertices have 
precisely $\lambda$ common neighbours. Note that a loop adds 1 to the degree of a vertex. Those $(v, k, \lambda)$-graphs which are loopless have been studied by Ahrens and Szekeres (1969), Bose and Shrikhande (1970), Rudvalis (1971) and Wallis (1969) among others.

We will assume throughout that all our matrices have order $n \times n$.

\section{The main theorem}

If $Q A R=A^{\mathrm{T}}$ for permutation matrices $Q$ and $R$, then $P(R A)=(R A)^{\mathrm{T}}$, where $P=Q R^{\mathrm{T}}$. Define $T(P)$ to be the set of all matrices $A$ such that $P(R A)=(R A)^{\mathrm{T}}$ for some permutation matrix $R$.

Let $A=\left(a_{i j}\right)$ and $B=\left(b_{i j}\right)$ be $n \times n$ matrices. Write $A \leqslant B$ if $a_{i j}=a_{k l}$ whenever $b_{i j}=b_{k l}$. Under the order $\leqslant$, the maximal elements of $T(P)$ will be called $P$-maximal. These matrices play a central role, as indicated in our first lemma.

LEMMA 2.1. If any P-maximal matrix is symmetrizable, then every matrix in $T(P)$ is symmetrizable.

We can now state our main result.

THEOREM 2.2. Consider the standard cyclic decomposition of the permutation corresponding to the permutation matrix $P$. Let $a_{1}, a_{2}, \ldots, a_{r}$ be the lengths of the even cycles and let $b_{1}, b_{2}, \ldots, b_{s}$ be the lengths of the odd cycles. Then every member of $T(P)$ is symmetrizable if and only if one of the following conditions is satisfied.

(1) $r=0$.

(2) $r=1$ and $\left(a_{1}, b_{i}\right)=1$ for $1 \leqslant i \leqslant s$.

(3) $r=2,\left(a_{1}, a_{2}\right)=2$ and $\left(a_{1}, b_{i}\right)=\left(a_{2}, b_{i}\right)=1$ for $1 \leqslant i \leqslant s$.

We will prove Theorem 2.2 by translating the problem into one which concerns the automorphism of a graph. Each $n \times n$ matrix $A=\left(a_{i j}\right)$ is associated with a graph $G(A)$. The vertices of $G(A)$ are the elements of the set $V_{1} \cup V_{2}$, where $V_{1}=\left\{r_{1}, r_{2}, \ldots, r_{n}\right\}$ and $V_{2}=\left\{c_{1}, c_{2}, \ldots, c_{n}\right\}$. The edges of $G(A)$ are the elements of $V_{1} \times V_{2}$, with each edge $\left(r_{i}, c_{j}\right)$ labelled (coloured) with the entry $a_{i j}$. Thus $G(A)$ is a complete bipartite edge-coloured graph.

For any $n \times n$ permutation matrix $S$, define $\pi(S)$ to be the permutation of $V_{1} \cup V_{2}$ which permutes $V_{1}$ according to $S$ and fixes each element of $V_{2}$. Let $\tau$ be the permutation $\left(r_{1} c_{1}\right)\left(r_{2} c_{2}\right) \ldots\left(r_{n} c_{n}\right)$. The various properties of $A$ that we have 
considered have simple interpretations in terms of the structure of $G(A)$, as we now describe

(1) $A$ is symmetric if and only if $\tau$ is an automorphism of $G(A)$.

(2) $A \in T(P)$ if and only if $\pi(R) \pi(P) \tau \pi(R)^{-1}$ is an automorphism of $G(A)$, for some $R$.

(3) $A$ is $P$-maximal if, for some $R$, the edge colours of $G(A)$ correspond exactly to the orbits of $V_{1} \times V_{2}$ induced by $\pi(R) \pi(P) \tau \pi(R)^{-1}$.

(4) $A$ is symmetrizable if and only if $\pi(S) \tau \pi(S)^{-1}$ is an automorphism of $G(A)$, for some $S$.

It is not hard to see that (2) and (4) imply the following two correspondences.

(1) $A$ is transposable if and only if there is an automorphism of $G(A)$ which swaps the sets $V_{1}$ and $V_{2}$.

(2) $A$ is symmetrizable if and only if there is an automorphism of $G(A)$ of order two which swaps $V_{1}$ and $V_{2}$.

In view of this translation, and of Lemma 2.1 , we can restate Theorem 2.3 as follows. Suppose that for some $R, \pi(R) \pi(P) \tau \pi(R)^{-1}$ is an automorphism of $G(A)$ and induces orbits of $V_{1} \times V_{2}$ corresponding exactly to the edge colouring of $G(A)$. Then $G(A)$ has an automorphism of order two interchanging $V_{1}$ and $V_{2}$ if and only if $P$ satisfies one of the conditions of Theorem 2.3.

By Lemma 2.1, we may assume that $\pi(R)$ is the identity. Write $\alpha=\pi(P)$ in terms of its elementary cycles as $\alpha=\alpha_{1} \alpha_{2} \ldots \alpha_{l}$, where $l=r+s$. Then $\alpha \tau$ has the cycle decomposition $\alpha \tau=\alpha_{1}^{*} \alpha_{2}^{*} \ldots \alpha_{1}^{*}$ where each elementary cycle $\alpha_{i}^{*}$ has twice the length of the corresponding $\alpha_{i}$. Let $G_{i}(A)$ be the subgraph of $G(A)$ induced by the vertices in the cycle $\alpha_{i}^{*}$. If the order of $G_{i}(A)$ is $2 n_{i}$, then the automorphism group of $G_{i}(A)$ is clearly the dihedral group of order $4 n_{i}$, in its standard representation of degree $2 n_{i}$.

We will label the vertices of $G(A)$ with pairs $(i, j)$, so that $\alpha_{i}^{*}$ is

$$
\left((i, 0),(i, 1), \ldots,\left(i, 2 n_{i}-1\right)\right)
$$

and

$$
(i, 0) \in V_{1} \text {, }
$$

for $1 \leqslant i \leqslant l$. Thus $(i, j)$ is in $V_{1}$ if $j$ is even and in $V_{2}$ if $j$ is odd. For each $i$, the second elements of the pairs $(i, j)$ are considered modulo $2 n_{i}$.

Any automorphism $\beta$ of order two of $G(A)$ which interchanges $V_{1}$ and $V_{2}$ can be written as $\beta=\beta_{1} \beta_{2} \ldots \beta_{l}$, where $\beta_{i}$ is an order two automorphism of $G_{i}(A)$. The 
possibilities for $\beta_{i}$ can be enumerated as follows.

'Type I. $\lambda_{i, m}$ maps $(i, j)$ to $(i, 4 m+1-j)$ for $1 \leqslant j \leqslant 2 n_{i}$.

Type II. $\mu_{i, m}$ maps $(i, j)$ to $(i, 4 m-1-j)$ for $1 \leqslant j \leqslant 2 n_{i}$.

Type III. (for $n_{i}$ odd) $v_{i}$ maps $(i, j)$ to $\left(i, n_{i}+j\right)$ for $1 \leqslant j \leqslant 2 n_{i}$.

It is easily seen that if $n_{i}$ is odd, $\lambda_{i, m}=\mu_{i, m+n_{i}-1}$ and $v_{i}=\left(\alpha_{i}^{*}\right)^{n_{i}}$.

We now consider the edges between $G_{i}(A)$ and $G_{j}(A)$ and seek $\beta_{i}$ and $\beta_{j}$ such that $\beta_{i} \beta_{j}$ preserves the colours of these edges. Note firstly that the edge $\left(i, u_{1}\right)\left(j, v_{1}\right)$ has the same colour as $\left(i, u_{2}\right)\left(j, v_{2}\right)$ if and only if there is an integer $t$ such that $u_{2} \equiv u_{1}+t\left(\bmod 2 n_{i}\right)$ and $v_{2} \equiv v_{1}+t\left(\bmod 2 n_{j}\right)$.

There are six cases to consider. We will examine the first case in detail, and then state the conclusion for the other five.

Case $(a) . \beta_{i}$ of type I, $\beta_{j}$ of type II.

Suppose $\beta_{i}=\lambda_{i, x}$ and $\beta_{j}=\mu_{j, y}$. Then $\beta_{i} \beta_{j}$ maps the edge $(i, u)(j, v)$ to the edge $(i, 4 x+1-u)(j, 4 y-1-v)$. These two edges have the same colour if and only if there is an integer $t$ such that

$$
4 x+1-u \equiv u+t\left(\bmod 2 n_{i}\right)
$$

and

$$
4 y+1-v \equiv v+t\left(\bmod 2 n_{j}\right)
$$

In order for $t$ to exist for any $u$ and $v$ of the opposite parity, it is necessary and sufficient that $\left(n_{i}, n_{j}\right) \leqslant 2$. If $u$ and $v$ have the same parity, there is no edge $(i, u)(j, v)$.

Case $(b) . \beta_{i}$ of type I, $\beta_{j}$ of type III $\left(n_{j}\right.$ odd).

Case (c). $\beta_{i}$ of type II, $\beta_{j}$ of type III ( $n_{j}$ odd).

Case $(d) . \beta_{i}$ of type $\mathrm{I}, \beta_{j}$ of type I.

Case (e). $\beta_{i}$ of type II, $\beta_{j}$ of type II.

In each of the cases above, analysis similar to that used in Case (a) shows that it is necessary and sufficient that $\left(n_{i}, n_{j}\right)=1$.

Case $(f)$. $\beta_{i}$ of type III, $\beta_{j}$ of type III ( $n_{i}$ and $n_{j}$ both odd).

Since $v_{i}=\left(\alpha_{i}^{*}\right)^{n_{i}}$ and $\left(\alpha_{i}^{*}\right)^{2 n_{i}}=1$, we see that $v_{i} v_{j}=\left(\alpha_{i}^{*} \alpha_{j}^{*}\right)^{n_{i n j}}$. Consequently $v_{i} v_{j}$ will always preserve the colour of the edges between $G_{i}(A)$ and $G_{j}(A)$.

In summary, it is necessary that $\left(n_{i}, n_{j}\right) \leqslant 2$, unless $n_{i}$ and $n_{j}$ are both odd. Now suppose that $n_{1}, n_{2}, \ldots, n_{r}$ are even, and $n_{r+1}, n_{r+2}, \ldots, n_{l}$ are odd.

(a) If $r=0$, we can use $\beta_{i}=v_{i}$ for $1 \leqslant i \leqslant l$. 
(b) If $r=1$, we must have $\left(n_{1}, n_{i}\right)=1$ for $2 \leqslant i \leqslant l$. In that case we can use $\beta_{1}=\lambda_{1,0}$ and $\beta_{i}=v_{i}$ for $2 \leqslant i \leqslant l$.

(c) If $r=2$, we must have $\left(n_{1}, n_{2}\right)=2$, and $\left(n_{1}, n_{i}\right)=\left(n_{2}, n_{i}\right)=1$ for $3 \leqslant i \leqslant l$. In that case we can use $\beta_{1}=\lambda_{1,0}, \beta_{2}=\mu_{2,0}$ and $\beta_{i}=v_{i}$ for $3 \leqslant i \leqslant l$.

(d) If $r \geqslant 3$, we must have $\left(n_{1}, n_{2}\right)=\left(n_{1}, n_{3}\right)=\left(n_{2}, n_{3}\right)=2$. However one of $\beta_{1}$ and $\beta_{2}$ must be of type I and the other of type II. Similarly, one of $\beta_{1}$ and $\beta_{3}$ must be of type I and the other of type II. Therefore $\beta_{2}$ and $\beta_{3}$ must be both of type I or both of type II, neither of which cases is allowed.

This completes the proof of Theorem 2.2 .

\section{Final remarks}

With the results of Section 2 we have a tool which can be used in certain circumstances to prove that a self-dual SBIBD is polar, given a correlation between the SBIBD and its dual. Since the binary matrices of order greater than three cannot be $P$-maximal for any $P$, no similar negative proof is possible.

It would be interesting to obtain classes of matrices for which transposability is sufficient to imply symmetrizability. This is not always true for binary matrices, as is shown by Everett and Metropolis (1972) with an example of order 16. A smaller example is shown in Figure 1.

At present the authors do not know of any self-dual SBIBD whose incidence matrix is not symmetrizable.

$$
\left[\begin{array}{llllllllllll}
1 & 1 & 0 & 0 & 1 & 1 & 1 & 0 & 1 & 0 & 0 & 1 \\
1 & 1 & 1 & 0 & 0 & 1 & 1 & 1 & 0 & 1 & 0 & 0 \\
1 & 1 & 1 & 1 & 0 & 0 & 0 & 1 & 1 & 0 & 1 & 0 \\
0 & 1 & 1 & 1 & 1 & 0 & 0 & 0 & 1 & 1 & 0 & 1 \\
0 & 0 & 1 & 1 & 1 & 1 & 1 & 0 & 0 & 1 & 1 & 0 \\
1 & 0 & 0 & 1 & 1 & 1 & 0 & 1 & 0 & 0 & 1 & 1 \\
1 & 0 & 0 & 1 & 0 & 1 & 1 & 0 & 0 & 0 & 0 & 1 \\
1 & 1 & 0 & 0 & 1 & 0 & 1 & 1 & 0 & 0 & 0 & 0 \\
0 & 1 & 1 & 0 & 0 & 1 & 0 & 1 & 1 & 0 & 0 & 0 \\
1 & 0 & 1 & 1 & 0 & 0 & 0 & 0 & 1 & 1 & 0 & 0 \\
0 & 1 & 0 & 1 & 1 & 0 & 0 & 0 & 0 & 1 & 1 & 0 \\
0 & 0 & 1 & 0 & 1 & 1 & 0 & 0 & 0 & 0 & 1 & 1
\end{array}\right]
$$




\section{References}

R. W. Ahrens and G. Szekeres (1969), 'On the combinatorial generalization of 27 lines associated with a cubic surface', J. Austral. Math. Soc. 10, 485-492.

R. C. Bose and S. S. Shrinkhande (1970), 'Graphs in which each pair of vertices is adjacent to the same number of other vertices', Studia Sci. Math. Hungar. 5, 181-195.

P. Dembowski (1968), Finite geometries (Ergebnisse der Math. und ihrer Grenzgebiete 44, SpringerVerlag, Berlin, Heidelberg, New York).

C. J. Everett and N. Metropolis (1972), 'On completely normal $(0,1)$-matrices and symmetrizability', $J$. Combinatorial Th. Ser. A. 13, 367-373.

A. Rudvalis (1971), ' $(v, k$, $\lambda$ )-graphs and polarities of $(v, k, \lambda)$-designs', Math. Z. 120, 224-230.

W. D. Wallis (1969), 'Certain graphs arising from Hadamard matrices', Bull. Austral. Math. Soc. 1, 325-331.

Department of Computer Science

University of Manitoba

Winnipeg, Manitoba R3T 2N2

Canada
Department of Mathematics

University of Melbourne

Parkville, Victoria 3052

Australia 